\title{
ANÁLISIS DEL BALANCE PRESUPUESTARIO DEL EJERCICIO FISCAL 2019 DEL PODER LEGISLATIVO DEL ESTADO DE VERACRUZ.
}

\section{ANALYSIS OF THE BUDGETARY BALANCE FOR FISCAL YEAR 2019 OF THE LEGISLATIVE BRANCH OF THE STATE OF VERACRUZ}

\author{
Dr. Eber Jardiel Pérez Zúñiga ${ }^{\text {a }}$ \\ Dr. Julio Alonso Iglesias ${ }^{\text {b }}$ \\ Mtro. Leonel Lara Serna ${ }^{c}$
}

${ }^{a}$ Universidad Veracruzana Facultad de Contaduría y Administración, uvenlinea@gmail.com ${ }^{\mathrm{b}}$ Universidad Veracruzana Instituto de Investigaciones en Contaduría, julalonso@uv.mx ${ }^{\mathrm{c}}$ Universidad Veracruzana Instituto de Investigaciones en Contaduría, leolara@uv.mx

\section{RESUMEN}

La presente investigación tiene como propósito analizar el Estado Analítico de Ingresos y el Estado Analítico del Ejercicio del Presupuesto de Egresos por el ejercicio fiscal 2019, se inicia con el análisis de las fuentes de financiamiento del ente para obtener los ingresos necesarios para su funcionamiento, posteriormente se analiza a través del Estado de Avance Presupuestal Por Objeto del Gasto al mes de diciembre de 2019 el ejercicio del gasto en sus diferentes momentos contables.

El objetivo del análisis es comparar el presupuesto estimado-recaudado con el presupuesto aprobado-ejercido, determinar a través de los indicadores de postura fiscal su balance presupuestario y dar a conocer los resultados de las clasificaciones administrativa funcional y económica en el seguimiento al ejercicio del gasto público lo que contribuye a consolidar la austeridad y erradicar la corrupción. Se realizó una investigación documental de información publicada en la plataforma de transparencia del ente fiscalizable y se comparó con la información publicada en la plataforma nacional de transparencia y las solicitudes de 
información enviadas. Se concluye que el ente presenta un balance presupuestario con superávit y sí cumplió con las disposiciones en el ámbito de sus atribuciones en términos de eficiencia, eficacia, racionalidad, austeridad y transparencia.

PALABRAS CLAVE: Ingresos; Egresos; Presupuesto; Superávit.

\section{ABSTRACT}

The purpose of this research is to analyze the Analytical State of Income and the Analytical State of the Exercise of the Expenditure Budget for fiscal year 2019, it begins with the analysis of the sources of financing of the entity to obtain the necessary income for its operation, subsequently Through the Budget Progress Statement by Object of Expenditure as of December 2019, the exercise of the expenditure is analyzed in its different accounting moments. The objective of the analysis is to compare the estimated-collected budget with the approved-exercised budget, determine through the fiscal position indicators its budget balance and present the results of the administrative, functional and economic classifications in the monitoring of the expenditure exercise public which contributes to consolidate austerity and eradicate corruption. A documentary investigation of information published on the transparency platform of the auditable entity was carried out and compared with the information published on the national transparency platform and the information requests sent. It is concluded that the entity presents a budget balance with a surplus and did comply with the provisions within the scope of its powers in terms of efficiency, effectiveness, rationality, austerity and transparency.

KEYWORDS: Income; Expenses; Budget; Surplus.

\section{INTRODUCCIÓN}

El presente trabajo de divulgación muestra los resultados de la investigación relacionada con el cumplimiento en términos de eficiencia, eficacia, racionalidad, austeridad y transparencia del poder legislativo del Estado de Veracruz en el ejercicio, control y evaluación del gasto público estatal para el ejercicio fiscal 2019. Se revisó el calendario de ministraciones del ejercicio fiscal 2019 del poder legislativo publicado en su portal de 
transparencia y se comparó con "El importe para el Poder Legislativo, que asciende a la cantidad de $\$ 776,116,100.00$ (Setecientos setenta y seis millones, ciento dieciséis mil cien pesos 00/100 M.N.)" Decreto 14 de 2018 [con fuerza de ley]. Por medio del cual se expide el Decreto de Presupuesto de Egresos. 28 de diciembre de 2018. Gaceta Oficial del Estado de Veracruz No. 520.

Se analizaron los ingresos por fuentes de financiamiento y se compararon con los estados analíticos de ingresos publicados por el ente (trimestral y anual) en la Plataforma Nacional de Transparencia, se identificaron y compararon los presupuestos; estimado, modificado (ampliaciones y reducciones) y recaudado, finalmente se determinó su diferencia. Se verificaron aritméticamente los avances presupuestales por objeto del gasto publicados en los portales de transparencia de los cuatro trimestres y de la presentación de cuenta pública anual del ejercicio fiscal 2019, no se encontraron diferencias en el presupuesto aprobado, la integración del presupuesto modificado es correcto conforme a las ampliaciones y reducciones entre las cuentas del mismo capítulo, se comprobaron los totales del presupuesto devengado, ejercido, pagado y el presupuesto sin devengar.

Con respecto al análisis del estado analítico del ejercicio del presupuesto de egresos la clasificación económica de tipo de gasto, gasto corriente y gasto de capital, son correctas conforme a las normas de la Ley General de Contabilidad Gubernamental. En resultado del balance presupuestario del ejercicio fiscal 2019 del poder legislativo del Estado de Veracruz a través del análisis de los indicadores de postura fiscal presenta un superávit por $\$ 26,769,849$ (Veintiséis millones setecientos sesenta y nueve mil ochocientos cuarenta y nueve pesos 00/100 M.N.).

El poder legislativo del Estado de Veracruz en materia presupuestaria para el año 2019, está obligado a operar conforme al Decreto de Presupuesto del Gobierno del Estado de Veracruz de Ignacio de la Llave de Egresos, la Ley de austeridad para el Estado de Veracruz de Ignacio de la Llave, Ley de Fiscalización Superior y Rendición de Cuentas del Estado de Veracruz de Ignacio de la Llave y la Ley de Ingresos del Gobierno del Estado de Veracruz de Ignacio de la Llave para el Ejercicio Fiscal 2020. 
El motivo principal de este análisis es para verificar el cumplimiento del presupuesto estimado-recaudado con el presupuesto aprobado-ejercido, determinar a través de los indicadores de postura fiscal su balance presupuestario y dar a conocer los resultados de las clasificaciones administrativa funcional y económica en el seguimiento al ejercicio del gasto público para determinar si el poder legislativo en el ejercicios fiscal 2019 cumplió y contribuyó a consolidar la austeridad, erradicar la corrupción y ejerció el prepuesto en términos de eficiencia, eficacia, racionalidad, austeridad y transparencia. Determinar el cumplimiento del poder legislativo del Estado de Veracruz en el ejercicio, control y evaluación del gasto público estatal para el ejercicio fiscal 2019 con las disposiciones en el ámbito de sus atribuciones en términos de eficiencia, eficacia, racionalidad, austeridad y transparencia. Revisar el calendario de ministraciones del ejercicio fiscal 2019 del poder legislativo publicado en su portal de transparencia y comparar con el decreto de presupuesto de egresos del gobierno del Estado de Veracruz de Ignacio de la Llave para el ejercicio fiscal 2019. Analizar los ingresos por fuentes de financiamiento y comparar con los estados analíticos de ingresos publicados trimestral y anualmente por el ente en la Plataforma Nacional de Transparencia, identificar los presupuestos; estimado, modificado (ampliaciones y reducciones) y recaudado, y determinar su diferencia. Analizar los avances presupuestales por objeto del gasto, trimestrales y anual, identificar el presupuesto aprobado, integrar el presupuesto modificado a través de las ampliaciones y reducciones, y comprobar los totales del presupuesto devengado, ejercido y pagado. Diferenciar en el estado analítico del ejercicio del presupuesto de egresos la clasificación económica de tipo de gasto, gasto corriente y gasto de capital. Determinar el déficit o superávit en el balance presupuestario del ejercicio fiscal 2019 del poder legislativo del Estado de Veracruz a través del análisis de los indicadores de postura fiscal.

\section{ANÁlisis DEL BALANCE PRESUPUESTARIO DEL EJERCICIO FISCAL 2019 DEL PODER LEGISLATIVO DEL ESTADO DE VERACRUZ.}

\section{1 Decreto del presupuesto de egresos}


"LA SEXAGÉSIMA QUINTA LEGISLATURA DEL HONORABLE CONGRESO DEL ESTADO LIBRE Y SOBERANO DE VERACRUZ DE IGNACIO DE LA LLAVE, EN USO DE LA FACULTAD QUE LE CONFIEREN LOS ARTÍCULOS 26 FRACCIÓN I INCISO a); 33 FRACCIÓN I Y 38 DE LA CONSTITUCIÓN POLÍTICA LOCAL; 18 FRACCIÓN I Y 47 SEGUNDO PÁRRAFO DE LA LEY ORGÁNICA DEL PODER LEGISLATIVO; 75 Y 77 DEL REGLAMENTO PARA EL GOBIERNO INTERIOR DEL PODER LEGISLATIVO; Y EN NOMBRE DEL PUEBLO, EXPIDE EL SIGUIENTE: DECRETO Número 14" Decreto 14 de 2018 [con fuerza de ley]. Por medio del cual se expide el Decreto de Presupuesto de Egresos. 28 de diciembre de 2018. Gaceta Oficial del Estado de Veracruz No. 520.

"El gasto total del Gobierno del Estado previsto en el presente Decreto, asciende a la cantidad de $\$ 128,361,911,179.00$ (Ciento veintiocho mil trescientos sesenta y un millones novecientos once mil ciento setenta y nueve pesos 00/100 M.N.), y corresponde al total de recursos aprobados en la Ley de Ingresos para el Estado de Veracruz de Ignacio de la Llave para el Ejercicio Fiscal 2019; dicha cantidad se distribuye conforme a lo que establece este capítulo y a sus diversas clasificaciones del gasto en los anexos presentados al final del presente Decreto.” Decreto 14 de 2018 [con fuerza de ley]. Por medio del cual se expide el Decreto de Presupuesto de Egresos. 28 de diciembre de 2018. Gaceta Oficial del Estado de Veracruz No. 520.

El importe para el poder legislativo asciende a la cantidad de $\$ 776,116,100.00$ (Setecientos setenta y seis millones ciento dieciséis mil cien pesos 00/100 M.N.), lo que representa el $0.6046 \%$ del total del presupuesto anual del Gobierno del Estado previsto para el Ejercicio Fiscal 2019, integrándose de acuerdo con los siguientes capítulos de gasto:

Tabla 1

Presupuesto integrado por capítulos de gasto.

\begin{tabular}{|lrr|}
\hline \multicolumn{1}{|c}{ Concepto } & Importe (Pesos) & \% \\
\hline Servicios Personales & $443,631,550.00$ & $57 \%$ \\
\hline Materiales y Suministros & $24,889,850.00$ & $3 \%$ \\
\hline
\end{tabular}




\begin{tabular}{|lrr|}
\hline Servicios Generales & $81,330,000.00$ & $10 \%$ \\
\hline Transferencias, Asignaciones, Subsidios y Otras Ayudas & $192,397,850.00$ & $25 \%$ \\
\hline Bienes Muebles, Inmuebles e Intangibles & $33,866,850.00$ & $4 \%$ \\
\hline \multicolumn{1}{c}{ Totales } & $\mathbf{7 7 6 , 1 1 6 , 1 0 0 . 0 0}$ & $\mathbf{1 0 0 \%}$ \\
\hline
\end{tabular}

Fuente: Decreto Número 14 (2018).

“La Comisión Permanente de Administración y Presupuesto del H. Congreso aprobará la distribución por concepto de gasto antes mencionado, en los términos señalados en la Ley Orgánica del Poder Legislativo del Estado de Veracruz de Ignacio de la Llave; asimismo, la rendición de cuentas y el resultado del ejercicio del gasto, se sujetarán a las disposiciones de dicho ordenamiento." Decreto 14 de 2018 [con fuerza de ley]. Por medio del cual se expide el Decreto de Presupuesto de Egresos. 28 de diciembre de 2018. Gaceta Oficial del Estado de Veracruz No. 520.

\section{2 Decreto del presupuesto de egresos}

Conforme al calendario de ministraciones del ejercicio fiscal 2019 del poder legislativo publicado en su portal de transparencia se comparó con el decreto de presupuesto de egresos del gobierno del Estado de Veracruz de Ignacio de la Llave para el ejercicio fiscal 2019 y se determinó que no existe ninguna solicitud de ampliación presupuestal.

Tabla 2

Calendario de ministraciones por el ejercicio fiscal 2019.

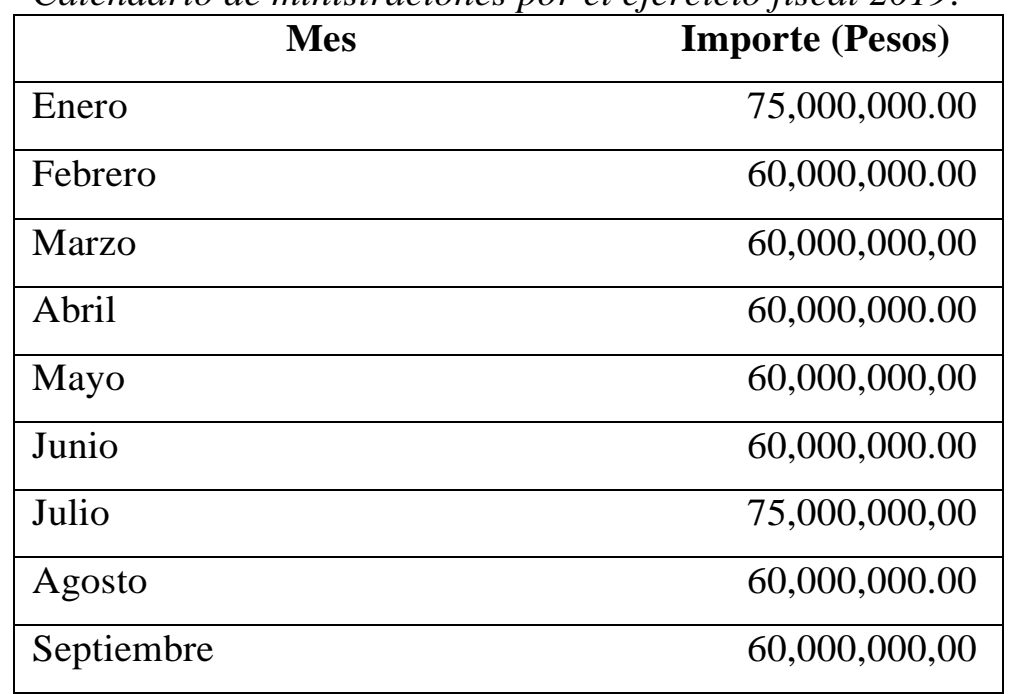




\begin{tabular}{|c|c|}
\hline Octubre & $65,000,000.00$ \\
\hline Noviembre & $60,000,000,00$ \\
\hline Diciembre & $81,116,100.00$ \\
\hline Total & $776,116,100.00$ \\
\hline
\end{tabular}

Fuente: Legisver (2020).

Conforme a los estados analíticos de ingresos trimestrales y anual del ejercicio fiscal 2019 publicados en la Plataforma Nacional de Transparencia, se identificaron ingresos por tres diferentes fuentes de financiamiento; Transferencias, asignaciones, subsidios y subvenciones y pensiones y jubilaciones; Productos (incluyen intereses que generan las cuentas bancarias de los entes públicos en productos) y además Ingresos por venta de bienes, prestación de servicios y otros (ingresos propios obtenidos por sus actividades diversas no inherentes a su operación que generen recursos y que son sean ingresos por venta de bienes o prestación de servicios, tales como donativos en efectivo, entre otros).

Conforme a los ingresos totales recaudados por el ente, el total publicado fue por $\$ 779,825,808$ (Setecientos setenta y nueve millones ochocientos veinticinco mil ochocientos ocho pesos 00/100 M.N.), mostrando una diferencia por $\$ 3,709,708$ (Tres millones setecientos nueve mil setecientos ocho pesos 00/100 M.N.) lo que representa un incremento del $0.4779 \%$ de presupuesto recaudado con respecto al presupuesto estimado a principio del ejercicio fiscal 2019, los ingresos presentan dos ampliaciones, por Productos la cantidad de $\$ 3,624,303$ (Tres millones seiscientos veinte cuatro mil trescientos tres pesos 00/100 M.N.) que representa un $0.4647 \%$ del total de los ingresos recaudados, y por Ingresos por venta de bienes, prestación de servicios y otros por la cantidad de $\$ 85,405$ (Ochenta y cinco mil cuatrocientos cinco pesos 00/100 M.N.) lo que representa un $0.0109 \%$.

Tabla 3

Estado analítico de ingresos del 1 de enero al 31 de diciembre de 2019.

\begin{tabular}{|lccrrrr|}
\hline Fuente de Financiamiento & Estimado & $\begin{array}{c}\text { Ampliacione } \\
\text { s y } \\
\text { Reducciones }\end{array}$ & Modificado & Devengado & Recaudado & $\begin{array}{c}\text { Diferen } \\
\text { cia }\end{array}$ \\
\hline $\begin{array}{l}\text { INGRESOS DEL PODER } \\
\text { LEGISLATIVO }\end{array}$ & $\mathbf{7 7 6 , 1 1 6 , 1 0 0}$ & $\mathbf{3 , 7 0 9 , 7 0 8}$ & $\mathbf{7 7 9 , 8 2 5 , 8 0}$ & $\mathbf{7 7 9 , 8 2 5 , 8 0}$ & $\mathbf{7 7 9 , 8 2 5 , 8 0}$ & $\mathbf{3 , 7 0 9 , 7 0}$ \\
$\mathbf{8}$ & & $\mathbf{8}$ & $\mathbf{8}$ \\
\hline
\end{tabular}




\begin{tabular}{|c|c|c|c|c|c|c|}
\hline Productos & 0 & $3,624,303$ & $3,624,303$ & $3,624,303$ & $3,624,303$ & $\begin{array}{r}3,624,30 \\
3\end{array}$ \\
\hline $\begin{array}{l}\text { Ingresos por venta de } \\
\text { bienes, prestación de } \\
\text { servicios y otros ingresos }\end{array}$ & 0 & 85,405 & 85,405 & 85,405 & 85,405 & 85,405 \\
\hline $\begin{array}{l}\text { Transferencias, } \\
\text { asignaciones, subsidios y } \\
\text { subvenciones y pensiones y } \\
\text { jubilaciones }\end{array}$ & $776,117,100$ & & $\begin{array}{r}776,116,10 \\
0\end{array}$ & $\begin{array}{r}776,116,10 \\
0\end{array}$ & $\begin{array}{r}776,116,10 \\
0\end{array}$ & \\
\hline Total & $\begin{array}{r}776,116,100.0 \\
0\end{array}$ & $3,709,708$ & $\begin{array}{r}779,825,80 \\
8\end{array}$ & $\begin{array}{r}779,825,80 \\
8\end{array}$ & $\begin{array}{r}779,825,80 \\
8\end{array}$ & $\begin{array}{r}3,709,70 \\
8\end{array}$ \\
\hline
\end{tabular}

Fuente: Legisver (2020).

\section{3 Egresos}

El presupuesto total para ejercer durante el ejercicio fiscal 2019 conforme al estado condensado de egresos se integró de la siguiente manera:

Tabla 4

Estado condensado de egresos por el ejercicio fiscal 2019.

\begin{tabular}{|cr|}
\hline \multicolumn{1}{|c|}{ Concepto } & \multicolumn{1}{c|}{$\begin{array}{c}\text { Importe } \\
\text { (Pesos) }\end{array}$} \\
\hline GASTOS DE FUNCIONAMIENTO & $\mathbf{5 4 9 , 8 5 1 , 4 0 0 . 0 0}$ \\
\hline SERVICIOS PERSONALES & $443,631,550.00$ \\
\hline MATERIALES Y SUMINISTROS & $24,889,850.00$ \\
\hline SERVICIOS GENERALES & $81,330,000.00$ \\
\hline TRANSFERENCIAS, ASIGNACIONES, SUBSIDIOS Y OTRAS AYUDAS & $\mathbf{1 9 2 , 3 9 7 , 8 5 0 . 0 0}$ \\
\hline SUBSIDIOS Y SUBVENCIONES & $180,484,105.00$ \\
\hline AYUDAS SOCIALES & $10,713,745.00$ \\
\hline DONATIVOS & $1,200,000.00$ \\
\hline ACTIVO BIENES MUEBLES PRESUPUESTALES & $\mathbf{3 3 , 8 6 6 , 8 5 0 . 0 0}$ \\
\hline ACTIVO BIENES MUEBLES PRESUPUESTALES & $33,866,850.00$ \\
\hline TOTAL DE GASTOS Y OTRAS PÉRDIDAS & $\mathbf{7 7 6 , 1 1 6 , 1 0 0 . 0 0}$ \\
\hline TOTAL & $\mathbf{7 7 6 , 1 1 6 , 1 0 0 . 0 0}$ \\
\hline
\end{tabular}

Fuente: Legisver (2020).

La clasificación por objeto del gasto (capítulo y concepto) muestra que un 96\% se aprobó para gasto corriente y solo un $4 \%$ para gasto de capital, conforme al Estado de avance presupuestal por objeto de gasto al 31 de diciembre de 2020, se determinó que el capítulo 2000 Materiales y Suministros presentó una reducción presupuestal por $\$ 8,102,949.40$ (32.56\% del total del presupuesto aprobado), el capítulo 3000 Servicios 
generales presentó una reducción presupuestal por \$8,832,120.22 (10.86\% del total del presupuesto aprobado), el capítulo 4000 Transferencias, asignaciones, subsidios y otras ayudas presentó una ampliación presupuestal por $\$ 43,251,317.51$ (22.48\% \% del total del presupuesto aprobado) y el capítulo 5000 Bienes Muebles, Inmuebles e Intangibles presentó una reducción presupuestal de $\$ 22,606,540.12$ (66.75\% del total del presupuesto aprobado).

\section{Tabla 5}

Presupuesto aprobado, ejercido y sin devengar por capítulos de gasto al 31 de diciembre de 2019.

\begin{tabular}{|lcrr|}
\hline \multicolumn{1}{|c}{ Concepto } & Aprobado & Ejercido & $\begin{array}{r}\text { Sin } \\
\text { devenga } \\
\text { r }\end{array}$ \\
\hline Servicios Personales & $443,631,550.00$ & $437,984,923.98$ & $\begin{array}{r}5,646,626 . \\
02\end{array}$ \\
\hline Materiales y Suministros & $24,889,850.00$ & $7,219,771.77$ & $\begin{array}{r}9,567,128 . \\
82\end{array}$ \\
\hline Servicios Generales & $81,330,000.00$ & $57,232,077.76$ & $\begin{array}{r}15,265,80 \\
2.02\end{array}$ \\
\hline $\begin{array}{l}\text { Transferencias, Asignaciones, } \\
\text { Subsidios y Otras Ayudas }\end{array}$ & $192,397,850.00$ & $235,649,167.51$ & 0.00 \\
\hline $\begin{array}{l}\text { Bienes Muebles, Inmuebles e } \\
\text { Intangibles }\end{array}$ & $33,866,850.00$ & $11,260,309.90$ & 0.00 \\
\hline \multicolumn{1}{c}{ Totales } & $\mathbf{7 7 6 , 1 1 6 , 1 0 0 . 0 0}$ & $\mathbf{1 0 0 \%}$ & \\
\hline
\end{tabular}

Fuente: Legisver (2020).

Conforme a la información presentadas en las Tablas 4 y 5 se determinó que el ente sí cumplió con las disposiciones en el ámbito de sus atribuciones en términos de eficiencia, eficacia, racionalidad, austeridad y transparencia. Es notorio que en tres de los cinco capítulos no se devengó el presupuesto aprobado (Ver Tabla 6), por lo que en términos de austeridad presenta un presupuesto sin devengar por $\$ 30,479,556.83$ si a eso le restamos la diferencia en ingresos por $\$ 3,709,708$ mostrados en la Tabla 3, obtenemos un balance presupuestario con superávit por $\$ 26,769,848.33$ (Ver Tabla 7).

\section{Tabla 6}

Presupuesto aprobado, modificado, devengado, sin devengar, ejercido y pagado por capítulos de gasto al 31 de diciembre de 2019.

\begin{tabular}{|lllll}
\hline Concepto & Aprobado & Modificado & Devengado & Sin devengar $\quad$ Pagado \\
\hline
\end{tabular}




\begin{tabular}{|c|c|c|c|c|c|c|}
\hline Servicios Personales & $443,631,550.00$ & $443,631,550$ & $437,984,923.98$ & $5,646,626.02$ & $437,984,923.98$ & $\begin{array}{r}428,306, \\
978.24\end{array}$ \\
\hline Materiales y Suministros & $24,889,850.00$ & $16,786,900.60$ & $7,219,771.78$ & $9,567,128.82$ & $7,219,771.77$ & $\begin{array}{r}7,112,77 \\
4.53\end{array}$ \\
\hline Servicios Generales & $81,330,000.00$ & $72,497,879.78$ & $57,232,077.76$ & $15,265,802.02$ & $57,232,077.76$ & $\begin{array}{r}50,205,6 \\
32.47\end{array}$ \\
\hline $\begin{array}{l}\text { Transferencias, } \\
\text { Asignaciones, Subsidios y } \\
\text { Otras Ayudas }\end{array}$ & $192,397,850.00$ & $235,649,167.51$ & $235,649,167.51$ & 0.00 & $235,649,167.51$ & $\begin{array}{r}235,649, \\
167.51\end{array}$ \\
\hline $\begin{array}{l}\text { Bienes Muebles, Inmuebles } \\
\text { e Intangibles }\end{array}$ & $33,866,850.00$ & $11,260,309.88$ & $11,260,309.88$ & 0.00 & $11,260,309.90$ & $\begin{array}{r}7,116,08 \\
8.68\end{array}$ \\
\hline Totales & $776,116,100.00$ & 779,825,807.77 & $749,346,250.94$ & $30,479,556.83$ & $749,346,250.93$ & $\begin{array}{r}728,390 \\
641.44\end{array}$ \\
\hline
\end{tabular}

Fuente: Legisver (2020).

\section{Tabla 7}

Indicadores de postura fiscal de la cuenta pública del ejercicio fiscal 2019.

\begin{tabular}{|c|c|c|c|}
\hline Concepto & Estimado & Devengado & Pagado \\
\hline Ingresos presupuestarios & $776,116,100$ & $776,116,100$ & $\begin{array}{r}776,116,1 \\
00\end{array}$ \\
\hline Egresos presupuestarios & $776,116,100$ & $749,346,251$ & $\begin{array}{r}728,390,6 \\
41\end{array}$ \\
\hline $\begin{array}{l}\text { Balance presupuestario } \\
\text { (Superávit) }\end{array}$ & $\mathbf{0}$ & $26,769,849$ & $\begin{array}{r}47,725,45 \\
9\end{array}$ \\
\hline
\end{tabular}

Fuente: Legisver (2020).

El balance presupuestario se presenta con superávit, el ente cumplió con las disposiciones en el ámbito de sus atribuciones en términos de eficiencia, eficacia, racionalidad, austeridad y transparencia.

\section{Figura 1}

Presupuesto sin devengar del ejercicio fiscal 2019.

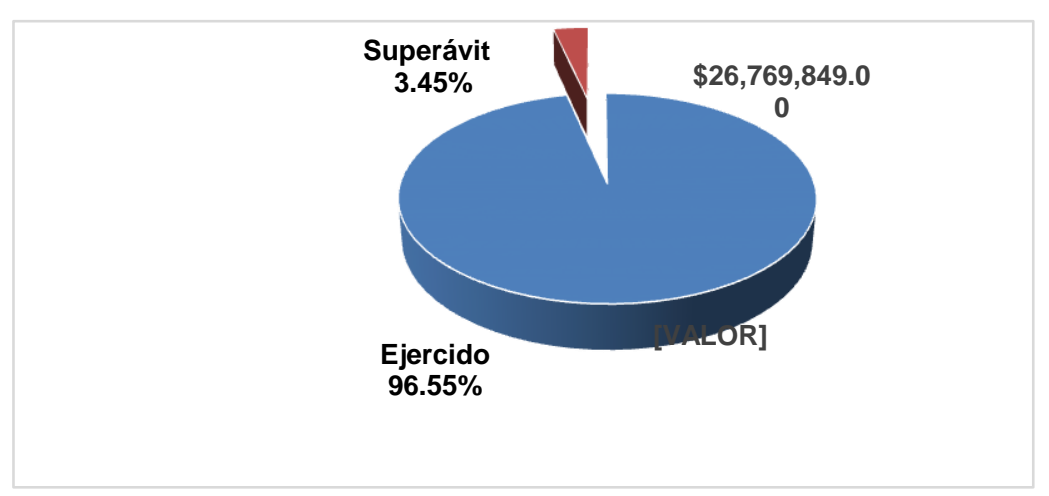

Fuente: Elaboración propia (2020).

Pérez E. J., Alonso J. \& Lara L.

Análisis del balance presupuestario del ejercicio fiscal 2019 del poder legislativo del estado de Veracruz. 


\section{METODOLOGÍA}

El estudio es de tipo comparativo, se realizó una investigación de tipo documental a través del análisis de la información publicada en los portales de transparencia del poder legislativo, del portal de transparencia de la Secretaría de Finanzas y Planeación, y de la Plataforma Nacional de Transparencia, además se realizaron solicitudes de información a través de los mismos portales de transparencia con la intención de confirmar que la información publicada corresponde en términos de transparencia y rendición de cuentas, se verificó aritméticamente la información presupuestaria y se comparó con la información contable presentada en los informes trimestrales y la cuenta pública anual del ente objeto de estudio. Se analizó que la información publicada por el ente cumpla con la normatividad vigente emitida por el Consejo Nacional de Armonización Contable y se encuentra disponible y actualizada.

\section{CONCLUSIONES}

El poder legislativo del Estado de Veracruz en el ejercicio, control y evaluación del gasto público estatal para el ejercicio fiscal 2019 cumplió con las disposiciones en el ámbito de sus atribuciones en términos de eficiencia, eficacia, racionalidad, austeridad y transparencia.

El poder legislativo recibió las ministraciones por parte de la Secretaría de Finanzas y Planeación por \$776, 116,600 conforme al calendario de ministraciones del ejercicio fiscal 2019 por concepto de Transferencias, asignaciones, subsidios y subvenciones y pensiones y jubilaciones, no existe solicitud de ampliación de presupuestal, por lo tanto, el ente cumplió con el decreto de presupuesto de egresos del gobierno del Estado de Veracruz de Ignacio de la Llave para el ejercicio fiscal 2019.

El ente obtuvo los ingresos por tres fuentes de financiamiento, el recaudado es mayor al estimado al generar ingresos por Productos (incluyen intereses que generan las cuentas bancarias de los entes públicos en productos) y además Ingresos por venta de bienes, prestación de servicios y otros (ingresos propios obtenidos por sus actividades diversas no inherentes a su operación que generen recursos y que son sean ingresos por venta de bienes o prestación de servicios, tales como donativos en efectivo, entre otros) por un total de 
$\$ 3,709,708$ (Tres millones setecientos nueve mil setecientos ocho pesos 00/100 M.N.) lo que representa recaudado mayor al estimado.

El poder legislativo durante el ejercicio fiscal 2019 cumplió con las disposiciones de racionalidad y austeridad al no devengar todo el presupuesto aprobado y presentar un balance presupuestario con superávit por $\$ 26,769,848.33$ conforme a los indicadores de postura fiscal.

Las ampliaciones y reducciones al presupuesto aprobado cumplen con la normatividad vigente emitida por el Consejo Nacional de Armonización Contable.

El poder legislativo podría operar con una disminución de su presupuesto hasta por 50, 000,000 de pesos para el ejercicio fiscal 2021, es decir, podría operar con un presupuesto de 725,000,000 de pesos para el ejercicio fiscal 2021, pero tendría que aumentar los recursos para Servicios personales en 15, 000,000 de pesos por el tema de cambio de legislatura y despido del personal de confianza.

El poder legislativo cumplió durante el ejercicio fiscal 2019 en términos de transparencia al contestar las solicitudes de información en forma oportuna y presentar en su portal como sujeto obligado la información presupuestaria conforme a la normatividad vigente.

\section{REFERENCIAS}

Congreso del Estado de Veracruz LXV Legislatura (2020). DECRETO DE PRESUPUESTO DE EGRESOS DEL GOBIERNO DEL ESTADO DE VERACRUZ DE IGNACIO DE LA LLAVE PARA EL EJERCICIO FISCAL 2019.

https://www.legisver.gob.mx/leyes/LeyesPDF/DPEGRESOS2019.pdf

Legisver (2020). Informes trimestrales de gastos cuarto trimestre 2019.

https://www.legisver.gob.mx/transparencia/FraccionXII/17_ClasificacionObjetoGasto4T.pdf

Plataforma Nacional de Transparencia (2020). Información financiera presupuestal y programática. https://consultapublicamx.inai.org.mx 
